\title{
An improved minimum variance beamforming applied to plane-wave imaging in medical ultrasound
}

Deylami, Ali Mohades; Asl, Babak Mohammadzadeh; Jensen, Jørgen Arendt

Published in:

Proceedings of 2016 IEEE International Ultrasonics Symposium

Link to article, DOI:

10.1109/ULTSYM.2016.7728895

Publication date:

2016

Document Version

Peer reviewed version

Link back to DTU Orbit

Citation (APA):

Deylami, A. M., Asl, B. M., \& Jensen, J. A. (2016). An improved minimum variance beamforming applied to plane-wave imaging in medical ultrasound. In Proceedings of 2016 IEEE International Ultrasonics Symposium IEEE. https://doi.org/10.1109/ULTSYM.2016.7728895

\section{General rights}

Copyright and moral rights for the publications made accessible in the public portal are retained by the authors and/or other copyright owners and it is a condition of accessing publications that users recognise and abide by the legal requirements associated with these rights.

- Users may download and print one copy of any publication from the public portal for the purpose of private study or research.

- You may not further distribute the material or use it for any profit-making activity or commercial gain

- You may freely distribute the URL identifying the publication in the public portal 


\section{An improved minimum variance beamforming applied to plane-wave imaging in medical ultrasound}

\author{
Ali Mohades Deylami \\ Department of Biomedical Engineering, \\ Tarbiat Modares University, \\ Tehran, Iran. \\ Ali.mohadesdeylami@modares.ac.ir \\ Joergen Arendt Jensen \\ Department of Electrical Engineering, \\ Technical University of Denmark, \\ Denmark \\ jaj@elektro.dtu.dk
}

\author{
Babak Mohammadzadeh Asl \\ Department of Biomedical Engineering, \\ Tarbiat Modares University, \\ Tehran, Iran. \\ babakmasl@modares.ac.ir
}

\begin{abstract}
Minimum variance beamformer (MVB) is an adaptive beamformer which provides images with higher resolution and contrast in comparison with non-adaptive beamformers like delay and sum (DAS). It finds weight vector of beamformer by minimizing output power while keeping the desired signal unchanged. We used the eigen-based MVB and generalized coherence factor (GCF) to further improve the quality of MVB beamformed images. The eigen-based MVB projects the weight vector with a transformation matrix constructed from eigen-decomposing of the array covariance matrix that increases resolution and contrast. GCF is used to emphasis on coherence part of images that improves the resolution. Four different datasets provided by IUS 2016 beamforming challenge are used to evaluate the proposed method. In comparison with DAS with rectangular weight vector, our method improved contrast about $8.52 \mathrm{~dB}$ and $6.20 \mathrm{~dB}$ for simulation and experimental contrast phantoms, respectively. It also enhanced lateral (axial) resolution about $87 \%(40 \%)$ and $\mathbf{7 3 \%}(\mathbf{2 1 \%})$ for simulated and experimental resolution phantoms, respectively.
\end{abstract}

Keywords-medical ultrasound imaging; adaptive beamforming; minimum variance beamformer; plane wave imaging

\section{INTRODUCTION}

Beamforming is a technique to form received echoes of an array of elements. The classical beamformer which is used in industrial medical ultrasound devices is delay and sum (DAS). This beamformer uses appropriate delays to synchronize the received backscattered echoes and then sums these delayed signals with multiplying by pre-defined weight vector. It seems necessary to move from ordinary non-adaptive beamformers to adaptive ones to improve the image quality. Adaptive beamformers find weight vector respect to received signal. So, each point has its own weight vector. Minimum variance beamformer (MVB) is one of the adaptive beamformer, finds the weight vector by minimizing the output power with maintaining the desired signal [1]. There are several methods presented for applying and improving performance of the MVB in medical ultrasound imaging [2-11]. Our goal in this paper is improving the image quality by combining the MVB with two other methods, eigen-based MVB [12] and generalized coherence factor (GCF) [13] to find the best low resolution image from one plane wave emission. The final high resolution image would be a sum of these low resolution images. The paper is organized as follows: the background section provides the necessary information for obtaining the delays and applying the MVB in medical ultrasound imaging. The Method section shows the combination of MVB with two other methods; eigen-based MVB and GCF methods. The results section presents the resulting images and measured metrics in comparison with ordinary DAS beamformer. Discussion and conclusion are provided at the last section.

\section{BACKGROUND}

In medical ultrasound imaging appropriate delays are applied to the received signals in time domain before multiplying them by the beamformer weight vector. In this paper, it is assumed that the array and imaging plane are located at $y$ plane (i.e. $y=0$ ). For an imaging point located at $\left[x_{i p}, z_{i p}\right]$ appropriate delay for a plane wave steered at $\theta_{i}$ and array element $m$ located at $\left[x_{a p_{m}}, z_{a p_{m}}\right]$ is obtained as follow:

$$
\begin{aligned}
& \tau_{m} \\
& =\frac{z_{i p} \cos \left(\theta_{i}\right)+x_{i p} \sin \left(\theta_{i}\right)}{c} \\
& +\frac{+\sqrt{\left(x_{i p}-x_{a p_{m}}\right)^{2}+\left(z_{i p}-z_{a p_{m}}\right)^{2}}}{c},
\end{aligned}
$$

where $c$ is the average speed of sound in the medium. The delayed signal received to $m$ th array element would be:

$$
s_{m}\left(x_{i p}, z_{i p}\right)=r_{m}\left(\tau_{m}\right),
$$

where $r_{m}$ is received signal by $m$ th element. For simplicity, the correspondence to imaging point will be omitted. Output of a $M$ elements array is obtained as follow: 


$$
u=\sum_{m=0}^{M-1} w_{m}^{\prime} s_{m}=\boldsymbol{w}^{H} \boldsymbol{s},
$$

where $\boldsymbol{w}$ is the array weight vector, $\boldsymbol{w}=\left[w_{0}, w_{1}, \ldots, w_{M-1}\right]^{T}$ and $\boldsymbol{s}$ is a vector of delayed received signals, $\boldsymbol{S}=\left[s_{0}, s_{1}, \ldots, s_{M-1}\right]^{T}$ and ${ }^{\prime},{ }^{T}, T$ stand for conjugate, Hermitian and transpose, respectively.

The MVB obtains the weight vector by minimizing the output power with maintaining the desired signal. The optimization problem of this beamformer can be written as follow:

$$
\min _{\mathbf{w}} E|u|^{2} \quad \text { subject to } \quad \boldsymbol{w}^{H} \boldsymbol{a}=1,
$$

where $\boldsymbol{a}$ is steering vector toward desired signal. By applying delays in time domain, it can be assumed that array always receives the desired signal from $0^{\circ}$, regardless of the steering angle of plane wave and location of imaging point. Therefore $\boldsymbol{a}$ becomes a vector of ones, i.e. $\boldsymbol{a}=[1,1, \ldots, 1]^{T}$. Using Lagrange multiplier, solution to the above optimization problem would be:

$$
\boldsymbol{w}_{M V}=\frac{R^{-1} \boldsymbol{a}}{\boldsymbol{a}^{H} R^{-1} \boldsymbol{a}}
$$

As seen, this beamformer needs the array covariance matrix. This matrix is not available because a limited number of samples is available. Therefore, a technique called spatial smoothing is used. In this way, the array is split to several overlapping subarrays and the covariance matrix is obtained by summing the covariance matrix of each subarray as follow:

$$
\hat{R}=\frac{1}{M-L+1} \sum_{l=0}^{M-L} \boldsymbol{s}_{l} \boldsymbol{s}_{l}^{H},
$$

where $\boldsymbol{s}_{l}$ is subarray with length $L$, $\boldsymbol{s}_{l}=\left[s_{l}, s_{l+1}, \ldots, s_{l+L-1}\right]^{T}$. In addition, it was shown that to improve the speckle quality, spatial averaging can be considered. In this way the estimated array covariance matrix would be the average of array covariance matrix in temporal $2 K+1$ samples as follow:

$$
\bar{R}=\frac{1}{2 K+1} \sum_{z=z_{i p}-z_{K}}^{z_{i p}+z_{K}} \hat{R}(z)
$$

Also, in order to increase robustness and numerical stability, a factor is summed to main diagonal of estimated array covariance matrix, which called diagonal loading as follow:

$$
\bar{R}_{d l}=\bar{R}+\Delta I .
$$

where $I$ is diagonal matrix.This factor usually is calculated from the estimated array covariance matrix as follow:

$$
\Delta=\delta \cdot \operatorname{trace}(\bar{R})
$$

where $\delta$ should be set manually. The final output of array is the sum of outputs of each subarray as follow:

$$
u=\frac{1}{M-L+1} \sum_{l=0}^{M-L+1} \boldsymbol{w}_{M V}^{H} \boldsymbol{s}_{l}=\boldsymbol{w}_{M V}^{H} \boldsymbol{s}_{s p}
$$

where $\boldsymbol{s}_{s p}=\frac{1}{M-L+1} \sum_{l=0}^{M-L+1} \boldsymbol{s}_{l}$.

\section{METHOD}

In order to further improve image quality, two different methods are investigated: Eigen-space based MVB and generalized coherence factor.

\section{A. Eigen-based MVB}

The aim of eigen-based MVB is to separate the signal space form noise space by decomposing the array covariance matrix in order to enhance the performance of the MVB. It projects the weight vector into a subspace constructed from the eigen vectors of the array covariance matrix. Firstly the array covariance matrix would be eigen-decomposed as follow:

$$
\bar{R}_{d l}=V \Lambda V^{H}
$$

where $\Lambda=\operatorname{diag}\left(\lambda_{0}, \lambda_{1}, \ldots, \lambda_{L-1}\right)$ in which $\lambda_{0} \geq \lambda_{1} \geq \cdots \geq$ $\lambda_{L-1}$ are the eigen values and $V=\left[\boldsymbol{v}_{0}, \boldsymbol{v}_{1}, \ldots, \boldsymbol{v}_{L-1}\right]$ is a matrix consists of corresponding eigen vectors. The signal subspace can be found as follow:

$$
E_{\text {signal }}=\left[\boldsymbol{v}_{0}, \boldsymbol{v}_{1}, \ldots, \boldsymbol{v}_{\text {num }}\right],
$$

and corresponding MVB weight vector in signal subspace would be:

$$
\boldsymbol{w}_{M V_{\text {signal }}}=E_{\text {signal }} E_{\text {signal }}^{H} \boldsymbol{w}_{M V} .
$$

num defines the number of kept eigen vectors. This number can be obtained in an adaptive manner. In this way we keep number of vectors that their cumulative sum of corresponding eigen values are greater than a predefined percentage of total sum of all eigen values.

\section{B. Generalized coherence facto}

Primary, generalized coherence factor $(\mathrm{GCF})$ is used to reduce the focusing errors resulting from wrong speed of sound estimation. However in further studies, it was shown that it can be used to more suppression of sidelobes. It finds how much the received signals are coherence by finding the ratio of signal energy of low-frequency region to the total energy. This factor is obtained as follow:

$$
G C F=\frac{\sum_{k \in \text { low frequency region }|p(k)|^{2}}}{\sum_{k=0}^{M-1}|p(k)|^{2}},
$$

where $p(k)$ is discrete Fourier transform (DFT) of the received array data along spatial domain. The low frequency region is considered as the $M_{0}$ samples of DFT around the zero frequency. The CGF is a value between 0 (non-coherence) and 1 (coherence). In this paper we use this factor to emphasize on 


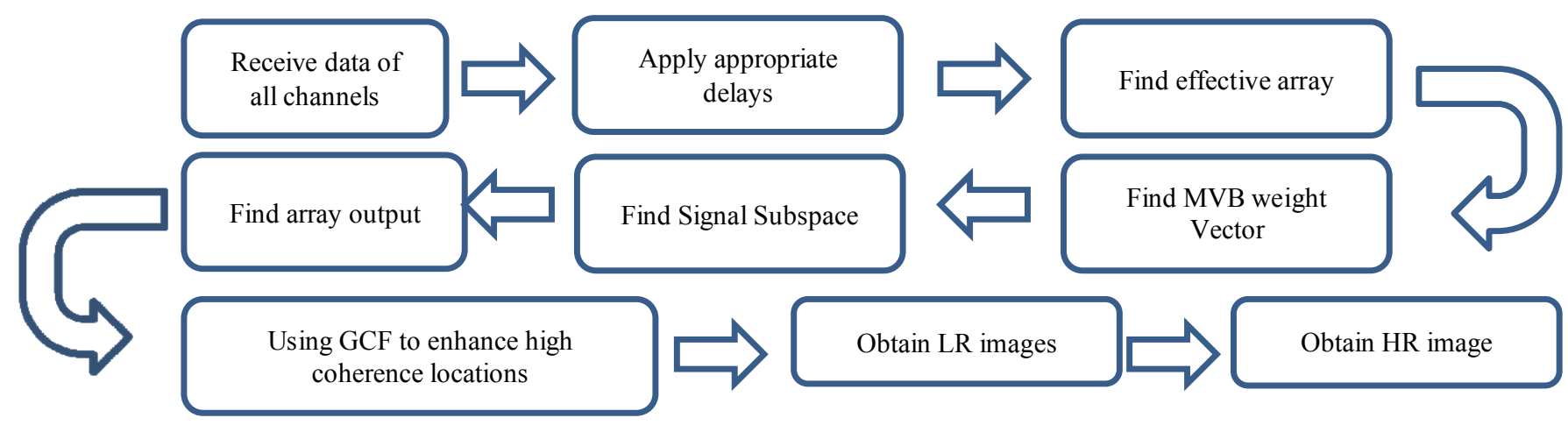

Fig. 1. Flowchart of the proposed method.

high coherence regions, which mostly are the location of high scattering regions as follow:

$$
u_{G C F}=u \times(1+G C F) .
$$

By adding 1 to GCF in the above equation, there is no concern about multiplying images by GCF around 0 .

It is worth to note that because of considering F\#, only a part of array element is considered for each point. In other words, the effective array size for each imaging point is defined by the F\# which is less or equal to $M$.

The Fig. 1 shows flowchart of the proposed method. For each plane wave, first we make a low resolution (LR) image for each emission $n=1,2, \ldots N_{e}$ and finally high resolution (HR) image for $N_{e}$ plane waves is obtained from LR images as follow:

$$
H R=\frac{1}{N_{e}} \sum_{n=1}^{N_{e}} L R(n) .
$$

\section{RESULTS}

In this section resulting images of four different datasets are presented. The datasets are provided by the IUS2016 beamforming challenge organizers. They considered two simulated and two experimental datasets, two of them are used to evaluate resolution and two are used for contrast. The metric are obtained based on evaluation procedure provided by the organizers of this challenge.

The resulting images are shown in Fig. 2. For each dataset also resulting images of ordinary DAS beamformer with rectangular window i.e. $\boldsymbol{w}_{D A S}=\frac{1}{M}[1,1, \ldots 1]^{T}$ are presented. In Table I, the parameters of proposed method are summarized. 75 plane waves with steering angles from $-16^{\circ}$ to $16^{\circ}$ are available. The images in Fig. 2 are the results of using just one plane wave steered at $0^{\circ}$ (emission number of 38 from 75). The measured contrast, axial and lateral resolution are demonstrated in Table II.
TABLE I. THE PARAMETERS USED IN THE PROPOSED METHOD.

\begin{tabular}{|c|c|c|}
\hline Parameter & $\begin{array}{c}\text { Resolution } \\
\text { Phantoms }\end{array}$ & $\begin{array}{c}\text { Contrast } \\
\text { phantoms }\end{array}$ \\
\hline$F \#$ & 1.5 & 1.5 \\
\hline$L:$ length of subarray & $\frac{M^{\mathrm{a}}}{2}$ & $\frac{M^{\mathrm{a}}}{2}$ \\
\hline$\delta$ : amount of diagonal loading & $1 \mathrm{e}-4$ & $1 \mathrm{e}-4$ \\
\hline $2 K+1$ : number of temporal samples & 1 & 11 \\
\hline $\begin{array}{c}\text { percentage of accumulative sum of } \\
\text { eigen values to total sum }\end{array}$ & $97 \%$ & $97 \%$ \\
\hline$M_{0}$ :number of samples to find GCF & 1 & 1 \\
\hline
\end{tabular}

${ }^{\text {a. }} M$ is the effective array length. It is defined by the $\mathrm{F} \#$ for each point independently.

TABLE II. CONTRAST AND RESOLUTION OBTAINED FROM EVALUATION PROCEDURE PROVIDED BY IUS 2016 BEAMFORMING CHALLENGE ORGANIZERS AND ALSO THE IMPROVEMENTS BY THE PROPOSED METHOD IN COMPARISON TO DAS FOR RESULTING IMAGES SHOWN IN FIG. 2.

\begin{tabular}{|c|c|c|c|c|}
\hline Beamformer & $\begin{array}{c}\text { Simulation } \\
\text { contrast } \\
\text { [dB] }\end{array}$ & $\begin{array}{c}\text { Experimental } \\
\text { contrast } \\
\text { [dB] }\end{array}$ & $\begin{array}{c}\text { Simulated } \\
\text { resolution } \\
\text { [mm] } \\
\text { Axial } \\
\text { Lateral }\end{array}$ & $\begin{array}{c}\text { Experimental } \\
\text { resolution } \\
\text { [mm] } \\
\text { Axial } \\
\text { Lateral }\end{array}$ \\
\hline DAS & 8.67 & 6.85 & $\begin{array}{c}0.40 \\
0.65\end{array}$ & $\begin{array}{c}0.57 \\
0.74\end{array}$ \\
\hline Proposed & 17.19 & 13.05 & 0.24 & 0.45 \\
Method & 8.52 & 6.20 & $40 \%$ & 0.20 \\
\hline Improvement & & & $87 \%$ & $21 \%$ \\
& & & & \\
\hline
\end{tabular}

\section{DISCUSSION AND CONCLUSION}

The resulting images of proposed beamformer, which is a combination of eigen-based MVB with GCF demonstrate that this technique can improve the resolution and contrast in comparison with the DAS. However the MVB showed improvement in image quality, further improvements are achieved by projecting the weight vector into signal space. By keeping $97 \%$ of energy received to array, we confirmed that approximately there is not any data loss with eigen-based MVB. Also, we used GCF to emphasis the coherence parts of image, which are mainly from high scattering regions. It improves lateral and axial resolutions. In ordinary coherencebased-methods, the output of array would be multiplied by that GCF, $u_{G C F}=G C F . u$. But problem with this multiplication occurs when the factor reaches to zero, specifically for the 


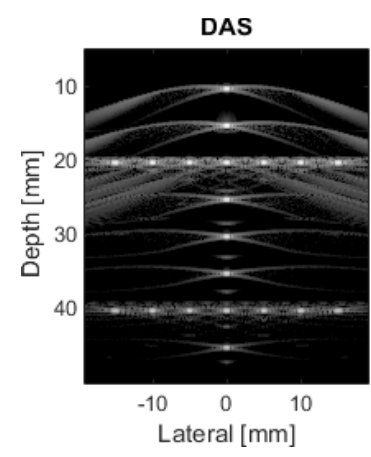

DAS

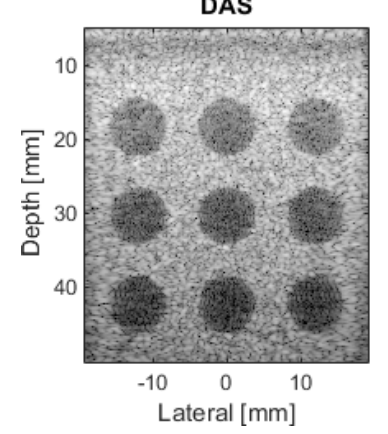

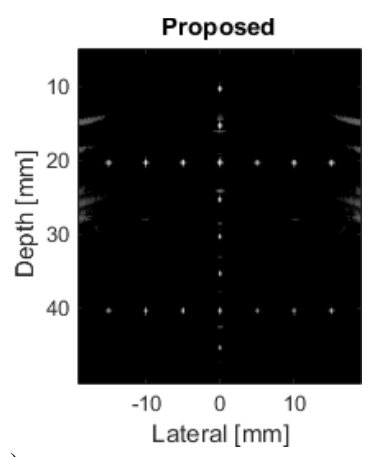

(a)

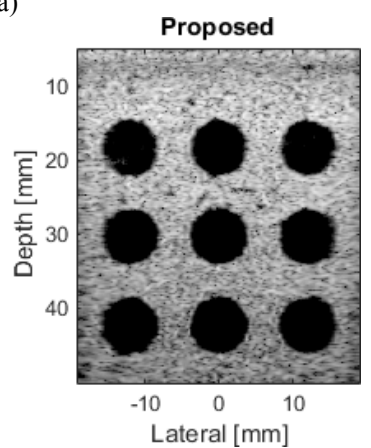

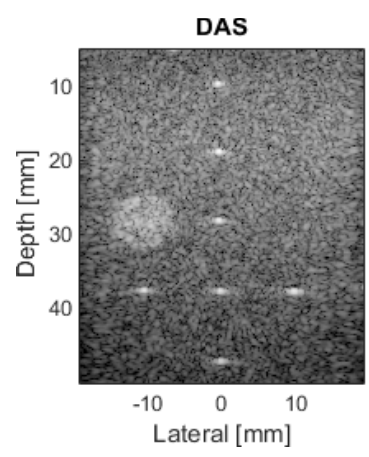

DAS

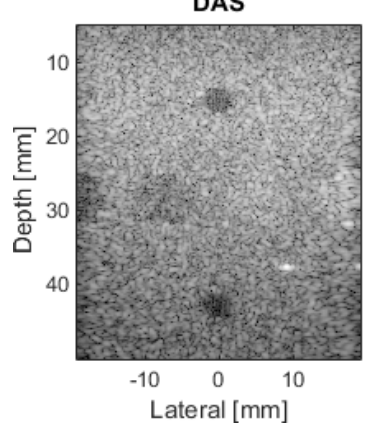

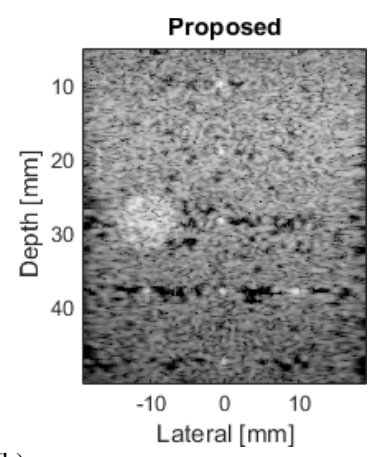

(b)

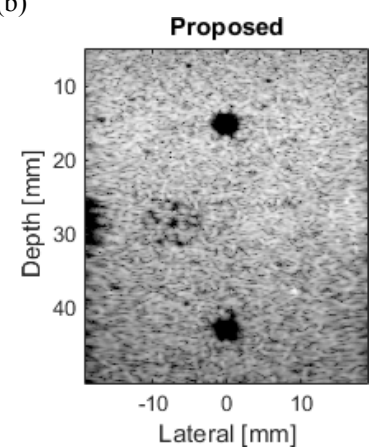

(d)

Fig. 2. Resulting images of the DAS and the proposed beamformers. (a) simulated resolution phantom (b) experimental resolution phantom (c) simulated contrast phantom (d) experimental contrast phantom. The images are the results of using just one plane wave steered at $0^{\circ}$.

speckles. We modified this factor by adding 1 to GCF, changing this factor from [0-1] to [1-2], which can be interpreted as $u_{G C F}=u+u * G C F$. Therefor the whole image is remained unchanged while the high coherence part would be emphasized. By the proposed method we find a low resolution image from one plane wave. To find the high resolution image, we sum up these low resolution images from different plane waves. However, we just show and compare the results of one emission.

\section{REFERENCES}

[1] J. Capon, "High-resolution frequency-wavenumber spectrum analysis," Proceedings of the IEEE, vol. 57, pp. 1408-1418, 1969.

[2] [2] C. C. Nilsen and S. Holm, "Wiener beamforming and the coherence factor in ultrasound imaging," Ultrasonics, Ferroelectrics and Frequency Control, IEEE Transactions on, vol. 57, pp. 1329-1346, 2010.

[3] [3] W. Zhisong, L. Jian, and W. Renbiao, "Time-delay- and timereversal-based robust capon beamformers for ultrasound imaging," Medical Imaging, IEEE Transactions on, vol. 24, pp. 1308-1322, 2005.

[4] [4] B. M. Asl and A. Mahloojifar, "Minimum variance beamforming combined with adaptive coherence weighting applied to medical ultrasound imaging," Ultrasonics, Ferroelectrics and Frequency Control, IEEE Transactions on, vol. 56, pp. 1923-1931, 2009.

[5] [5] B. M. Asl and A. Mahloojifar, "Contrast enhancement and robustness improvement of adaptive ultrasound imaging using forwardbackward minimum variance beamforming," Ultrasonics, Ferroelectrics and Frequency Control, IEEE Transactions on, vol. 58, pp. 858-867, 2011.
[6] [6] J. F. Synnevaag, A. Austeng, and S. Holm, "Benefits of minimumvariance beamforming in medical ultrasound imaging," Ultrasonics, Ferroelectrics and Frequency Control, IEEE Transactions on, vol. 56, pp. 1868-1879, 2009.

[7] [7] A. C. Jensen and A. Austeng, "An approach to multibeam covariance matrices for adaptive beamforming in ultrasonography," Ultrasonics, Ferroelectrics and Frequency Control, IEEE Transactions on, vol. 59, pp. 1139-1148, 2012.

[8] [8] I. K. Holfort, F. Gran, and J. A. Jensen, "Broadband minimum variance beamforming for ultrasound imaging," Ultrasonics, Ferroelectrics, and Frequency Control, IEEE Transactions on, vol. 56, pp. 314-325, 2009.

[9] [9] F. Viola and W. F. Walker, "Adaptive signal processing in medical ultrasound beamforming," in IEEE Ultrasonics Symposium, 2005., 2005, pp. 1980-1983.

[10] [10] M. Sasso and C. Cohen-Bacrie, "medical ultrasound imaging using the fully adaptive beamformer," in Proceedings. (ICASSP '05). IEEE International Conference on Acoustics, Speech, and Signal Processing, 2005., 2005, pp. 489-492.

[11] [11] J. A. Mann and W. F. Walker, "A constrained adaptive beamformer for medical ultrasound: initial results," in Ultrasonics Symposium, 2002. Proceedings. 2002 IEEE, 2002, pp. 1807-1810 vol.2.

[12] [12] B. M. Asl and A. Mahloojifar, "Eigenspace-based minimum variance beamforming applied to medical ultrasound imaging," Ultrasonics, Ferroelectrics and Frequency Control, IEEE Transactions on, vol. 57, pp. 2381-2390, 2010.

[13] L. Pai-Chi and L. Meng-Lin, "Adaptive imaging using the generalized coherence factor," Ultrasonics, Ferroelectrics and Frequency Control, IEEE Transactions on, vol. 50, pp. 128-141, 2003. 